\title{
Assessment method for supply chain benefits
}

\author{
Fernando Cezar Leandro Scramim ${ }^{1}$ and Mário Otávio Batalha ${ }^{2}$ \\ ${ }^{1}$ Department of Production Engineering, São Carlos Federal University, UFSCar, Via Washington Luiz, Km 235, CEP 13565-905 \\ São Carlos, São Paulo, Brazil, fernandosc@fsa.br \\ ${ }^{2}$ Department of Production Engineering, São Carlos Federal University, UFSCar, Brazil
}

\begin{abstract}
Competitiveness in an agrichain is associated with efficient internal organization and with the communication systems and coordination of activities between companies in the chain. The objective of this paper is to describe how the system dynamics (SD) methodology can help the re-formulation processes of economic and productive systems, while also evaluating new interventionist policies. The SD methodology seeks to model each of the casual links explicitly, and to track the resulting system behavior over time. In this paper, a network of companies in the Brazilian milk supply chain is defined in terms of an SD model. In particular, it shows that before actions are taken, their impacts on the agent's production costs can be forecasted, giving more consistency to the study. Three scenarios are developed to study the system behavior over the simulation time by varying the expected performance of the technical assistance service introduced in the network. The network is composed of three representative groups of rural producers and a dairy company. Input data are based on a network in the central region of the state of São Paulo. The agents' unit costs are forecasted to decrease after coordinated actions are taken throughout the network over the simulation time. As a result, non-negative operational profit margins can be reached giving more stability to the whole system.
\end{abstract}

Keywords: Supply chain coordination; production costs; Brazilian milk industry, operational performance

\section{Introduction}

The study presented in this paper focuses on applying the system approach to problems affecting agribusiness. In this approach, management competition demands internal and inter-organizational efficiency. Thus, it is necessary to associate competitiveness with efficient internal organization and with the communication systems and coordination of activities between companies in an agrichain. The application of the concepts of Supply Chain Management (SCM) is considered here as an alternative to improve the performance of agrichains (Cooper et al., 1997; Sonka and Cloutier, 1998). SCM's goal is to promote mutual benefits between the agents of a system through the definition of organizational structures and contractual relationships. In this study these concepts are assumed to be very helpful in the re-formulation process of economic and productive systems.

Ballou et al. (2000) point out that the most challenging situation occurs when the benefits reside ("pool") with some members to the detriment of others in an agricultural supply chain. Balancing these benefits so that all members are better off for their cooperation is the new challenge for supply chain managers. The authors point out that with an SCM approach new metrics tool ("accounting systems") should be able to define and measure costs and other relevant data among the various channel members.
The case of the Brazilian milk supply chain is specifically considered in this paper because of its large potential for application of the system approach and the SCM concept. Although Brazil is the third-largest milk producer in the world with 19 billion liters/year, its productivity is one of the worst. The milk industry in Brazil is composed of a large number of small-scale farm operators, with inefficient production methods, low integration with dairy companies and no long-term plans for increasing productivity in the whole system. Regional dairy companies are facing technological pressure caused by the installation and the acquisition process of large-scale multinational companies that has taken place during the last decade. As a result, the regional dairy companies and farmers are now addressing a lack of financial return on their investments. Thus, a future quality and quantity crisis in the sector can be foreseen, unless private and public action is taken to achieve better firm performance (farm operators and dairy companies) and performance of the whole system.

The network configuration, together with joint action, has become an alternative way to confront the challenges imposed in this competitive situation. For farm operators (rural producers), the major prerequisite to achieving better economic performance and increasing milk production is surely increased productivity. Silva (2000) estimates that a farm's productivity increases an average of three percent 
per year in Brazil. The author points out that the greater concentration of dairy processing among large multinational companies, such as Nestlé and Parmalat, is stimulating Brazilian dairy farmers to invest in genetics, nutrition, and management practices. For example, the author cites that between 1996 and 2000 the semen market in Brazil increased by more than 30 percent. However, given the low investment capacity of the majority of small-scale and technologically old-fashioned farm operators it is also necessary to find low-cost solutions, which can have a significant impact on their economic performance and competitiveness. This would also discourage the migration of rural workers to the cities, thereby avoiding the associated social costs and decreases in potential rural production throughout the country.

In view of the risks and new opportunities in this Brazilian context, we assume that the operation of a network (dairy industry and rural producers), following the principles of SCM, can generate mutual benefits for the agents and improve the performance of the global system. This can be achieved by integrating the functions of production planning, production costs and logistics costs optimization. The provision of technical assistance to the members of the network is also needed. Ballou et al. (2000) introduced the question of whether these potential benefits, whether or not they actually exist, can be measured. We propose in this study that the use of the system dynamics simulation approach could be very useful in attempting to validate SCM concepts, working as a metric tool to define and measure costs and other relevant data among the channel members. The SD modeling in the Brazilian milk supply chain presented in this paper is intended to contribute to a new metric measuring system in which the impact of new policies can be forecasted before their implementation, and the benefits of cooperation can be estimated. It can also function during the implementation process as a feedback and control mechanism.

The objective of this paper is to describe how SD methodology can help managers analyze alternatives in the re-formulation process of economic and productive systems. A network of companies in the Brazilian milk supply chain is defined in terms of an SD model. The paper begins with an introduction of the SD method and discusses some applications of this method in the literature. The third part of this work presents a description of the elements of the model as well as its casual inter-relations (network information) in the Brazilian milk supply chain. Three scenarios are then developed to study the system behavior over the simulation time by varying the expected performance of the technical assistance service introduced into the network. Finally, the potential of the SD methodology is discussed.

\section{System dynamics - method and practice}

The SD method comes from the discipline of systems thinking. Systems thinking is a school of thought that offers a way to communicate about dynamic complexities and interdependencies. It aims to represent circular or mutually causative relationships rather than linear causal ones (Anderson and Johnson, 1997). The main practical result of systems thinking is the identification of the systems' leverage points. These leverage points are areas where small changes in the system's structure can yield significant and lasting improvements to the whole system (Senge, 1994) . The SD model is a methodology for constructing computer simulation models to provide a theoretical and empirical base for structuring the relationships in complex systems. The SD structure is formalized via a simulation model, which essentially consists of two components: the stock and flow network and the information network. Mathematically, an SD model views the world as a set of differential equations. The prediction of the system state at any future point is obtained by integration, starting from the initial state (Buongiorno, 1996). The SD methodology seeks to model each of the casual links explicitly, and to track the resulting system behavior over time.

A few software packages were developed for Windows interface to facilitate the use and understanding of the SD method. By using various types of icons and defining the connections between them, we can represent in these computer programs the entire structure of a system. Connecting the icons generates the integral equations. The computer does all the integrating and the only math that has to be done manually involves addition, subtraction, multiplication, and division. For every icon in a model, there is an equation that defines it (Cover, 1996).

Several SD models presented in the literature during the last decade were developed to study economic and social systems. Buongiorno (1996) used system dynamics with econometrics to predict capacity changes in the industrial forestry sector. Yonenaga (2001) applied SD modeling in forest management to determine the best moment to cut a forest for supplying the wood-pencil industry. Saito (2000) developed a SD model to identify leverage points in the Brazilian sugar-cane supply chain. Smith and van Ackere (2002) arranged within an SD model a traditional economic model of supply and demand for the British National Health Service.

Sonka and Cloutier (1998) applied SD modeling to explore the economic role of information feedback in the strategic management of physical and financial stock and flow interactions within the pork production/packing interface in the pork sector in the United States. Conceptualized with 
the input of industry participants, the hog production and packing value chain model sought to capture physical, economic and conformance feedback interactions in the executions of activities over time.

Fisher, D. K. et al. (2000) utilized a system dynamics model to explore the adoption and diffusion of yield monitoring and mapping technology by soybean producers in the United States. The model intended to make explicit the causal relationships that influence technology adoption and diffusion behavior. The authors point out that managers in agricultural businesses are faced with a dynamic, complex, and uncertain environment in which to make decisions. The factors affecting decision outcomes change over time, and results are not known at the time decisions are made. Learning about the consequences of different decision options should improve managers' performance. SD modeling is one type of management tool that can help in this process.

These related simulation approaches were intended to allow members of the agribusiness community to gain insight into the causal factors influencing supply chain agents' decision-making processes and thereby into the potential diffusion patterns resulting from those processes. However, the shortcomings pointed out in Ballou et al. (2000) are still present. Firstly, a difficult situation can arise in which the benefits reside ("pool") with some members to the detriment of others. Secondly, the real benefits of cooperation cannot be estimated or known in advance.

\section{System dynamics modeling}

For this study, a milk reception and processing network was defined, with a given productive capacity of the dairy company and three groups of rural producers. This model is intended to represent the current conditions of coordination and organizational structure between economic agents (dairy companies and rural producers) in the Brazilian milk industry. The input data are based on the real cost structure of each group of rural producers represented in the model as well as the average medium-scale dairy company cost structure in the central region of the state of São Paulo.

We illustrate this work with the software Stella ${ }^{\circledR} 5.0$ (STELLA and STELLA Research, 1997). The stock and flow network and the information network of the model are shown in Appendix A. There are three stocks in the model, indicated by rectangles ("Received Vol. F1", "Received Vol. F2", and "Received Vol. F3"). They represent the amount of (verified quality) milk the dairy company receives each month. The in-flows of each "stock" represent the monthly production of each group of rural producers; these are represented in the model by the variable flows "Monthly production 1",
"Monthly production 2", and "Monthly production 3". The first group of rural producers is composed of 80 mediumscale dairy farms with a 500-liters/day average production. The second group is composed of 15 large-scale dairy farms, with a 1000-liters/day average production. The third group comprises the majority of the rural producers in Brazil: 200 small-scale and low-productivity dairy farms, with a 100liters/day average production. This is the usual classification for milk producers in Brazil. The average production of the whole network represented here is 254-liters/day by each rural producer. This structure is very similar to the real situation of the dairy farms in Brazil. For example, in 2000, private dairy companies like Nestle and Parmalat had respectively 270 and 162-liters/day average milk production in the rural producers' network (Leite Brasil, CNA/Decon, Embrapa, 2000).

The monthly production of each group of rural producers is altered by the seasonal factor (variable "Seasonality" in the model). Seasonality refers to the winter season in Brazil when milk output is reduced because of the lack of rain (dry season). The monthly production of each group of farms is also changed by the installation, in the 6th month of the simulation, of a technical assistance agent (variable "Tech Asst Imp" in the model). The basic function of the technical assistance agent is supposed to be the global planning of the network. This planning should be in terms of volume projections ("Tech Asst Imp") and the expected quality in the raw material (milk) over time. The expected increase in the raw material quality is represented in the model by the variable "Refusal rate", which is supposed to decrease over time after the introduction of the technical assistance agent. Table 1 lists the model variable abbreviations and their meanings.

Several activities must be performed to help the agents achieve better quantity and quality of performance. Global breeding plans that do not interfere with the supply of the dairy industry, sanitary control of the animals, pasture studies to improve the farm performance (productivity), and the exchange of technological innovations are examples of the activities that can be performed by the technical assistance agent. This leads to the principles of Supply Chain Management: the integration of processes (operational and strategic activities) to improve the global system performance. To be able to perform the proposed functions, the technical assistance agent should be composed of qualified professionals (technical and management features). A lot of organizational configurations could be proposed for that agent. However, this discussion would go beyond the limits of this paper.

Of course, introduction of a technical assistance agent should be followed by an increase in the system costs. Moreover, the total technical assistance agent cost has to 
Table 1. List of the model variables and their meanings.

Variables

Received Vol. F1

Received Vol. F2

Received Vol. F3

Monthly production 1

Monthly production 2

Monthly production 3

Flow F1

Flow F2

Flow F3

Waste F1

Waste F2

Waste F3

DC Processing

Seasonality

Tech Asst Imp

Fc1 (Fcu1)

Fc2 (Fcu2)

Fc3 (Fcu3)

Vcu1

Vcu2

Vcu3

F1Tac (F1TAcu)

F2Tac (F2TAcu)

F2Tac (F3TAcu)

NQcu1

NQcu2

NQcu3

Tcu1

Tcu2

Tcu3

Profit1u

Profit2u

Profit3u

Price DCRM

Refusal Rate

FcDC (FcuDC)

DCTAC (DCTACU)

VCURMDC

Other $\mathrm{VCDC}$

TcuDC

\section{Meaning}

Amount of milk supplied each month by medium-scale producers

Amount of milk supplied each month by large-scale producers

Amount of milk supplied each month by small-scale producers

The monthly production flow of medium-scale rural producers

The monthly production flow of large-scale rural producers

The monthly production flow of small-scale rural producers

Medium-scale producers' flow of milk processed by Dairy Company

Large-scale producers' flow of milk processed by Dairy Company

Small-scale producers' flow of milk processed by Dairy Company

Medium-scale producers' flow of milk refused by Dairy Company

Large-scale producers' flow of milk refused by Dairy Company

Small-scale producers' flow of milk refused by Dairy Company

Total flow of milk processed by Dairy Company

Seasonal factor on the rural producers' monthly outcome

Increase in the farms' productivity by the technical assistance agent

Total fixed cost of medium-scale rural producers (fixed unit cost)

Total fixed cost of large-scale rural producers (fixed unit cost)

Total fixed cost of small-scale rural producers (fixed unit cost)

Variable unit cost of medium-scale rural producers

Variable unit cost of large-scale rural producers

Variable unit cost of small-scale rural producers

Technical assistance cost assumed by medium-scale rural producers (unit cost)

Technical assistance cost assumed by large-scale rural producers (unit cost)

Technical assistance cost assumed by small-scale rural producers (unit cost)

Non-quality unit cost of medium-scale rural producers

Non-quality unit cost of large-scale rural producers

Non-quality unit cost of small-scale rural producers

Total unit cost of medium-scale rural producers

Total unit cost of large-scale rural producers

Total unit cost of small-scale rural producers

Unit profit margin of medium-scale rural producers

Unit profit margin of large-scale rural producers

Unit profit margin of small-scale rural producers

Price paid by Dairy company for the milk supplied

Refusal rate of the received milk

Dairy company's fixed cost (fixed unit cost)

Technical assistance cost assumed by Dairy company (unit cost)

Dairy Company's variable cost (raw material)

Others Dairy company's variable costs

Dairy company's total unit cost be shared between the model's agents ( 3 groups of rural processors and the dairy company). Here it is assumed that this cost of technological assistance could be shared as follows: one sixth to each group of producers and half to the dairy company. In fact, this is not the most appropriate way to share this cost throughout the system. The effect of the technical assistance in each element of the network should be exactly projected. For this study, the improvement that can be achieved through the introduction of the technical assistance service, represented by the variable "Tech Asst 
Imp", is considered to be the same for all groups of farms as well as the final behavior of the variable "Refusal rate" over the simulation time. The main benefit to the agents and to the whole system is a reduction in the unit costs over time through better performance in quality and quantity indicators despite the incorporation of the technical assistance service cost throughout the system.

The total unit cost of each group of rural producers ("Tcu1", "Tcu2", and "Tcu3") is defined following the principles of the direct costing method (Horngren and Foster, 1991). Thus, the total unit cost of each group of dairy farms is composed of the unit cost of the network technical assistance ("F1TAcu", "F2TAcu", and "F3TAcu"), fixed unit cost ("Fcu1", "Fcu2", and "Fcu3"), the average variable unit cost ("Vcu1", "Vcu2", and "Vcu3"), and the non-quality unit cost ("Nqcu1", "Nqcu2", and "Nqcu3"). The average fixed unit cost is obtained by dividing the total fixed cost of each group of rural producers ("Fc1", "Fc2", and "Fc3") by the respective monthly milk production. The average variable unit cost represents the part of the milk unit cost that is not influenced by the monthly production; i.e., regardless of how many liters of milk are produced this unit cost will be the same, unless technology changes (e.g., the labor cost in the farms' and dairy company's production process). If technology changes for reduction in variable unit cost, it normally results in an increment of fixed cost (e.g. equipment depreciation cost). The last component of the total cost is the non-quality unit cost, which is obtained by multiplying the refusal rate ("Refusal rate") by the price that the dairy company would pay for the adequate raw material ("Price DCRM"). This component represents the cost of revenue loss. Once refused at the dairy company reception plant, the produced and delivered raw material has no way to become useful again.

Several assumptions had to be made to run the model. The first one is related to the price of the milk (raw material for the process) and its oscillation throughout the simulation time. The price paid for the raw material is projected to increase over the simulation time. A less strong price oscillation is also projected between the dry and normal seasons. These assumptions can work as an incentive mechanism in order to avoid network volume reduction during the dry season when some rural producers can be attracted by other companies' offers (opportunistic behavior). Moreover, this increase in the milk price can also be assumed because seasonality is expected to have less influence seasonality on the dairy farms' quantity performance over time. Usually this price is a result of the offer and demand equilibrium. In the dry season the price tends to rise and during the summer season, when the production is higher, the price is reduced again. For example, the average price of type " $\mathrm{C}$ " fluid milk paid to producers during 2000 reached US $\$ 0.17$ per liter, up 21 percent from US $\$ 0.14$ per liter in 1999 . The peak of higher prices paid to producers occurred (US\$ $0.21 /$ liter) during the months of July and August 2000 (Silva, 2000), which is the winter season in the southern hemisphere. Figure 1 shows the projected price and the expected seasonal influence over the simulation time. The lowest price (during the summer months) is projected to rise from US\$ 0.15 (initial condition) to about US $\$ 0.18$ over the last two projected summer seasons.

The dairy company's total unit cost is similarly composed of the fixed unit cost ("FcuDC"), variable raw-material unit

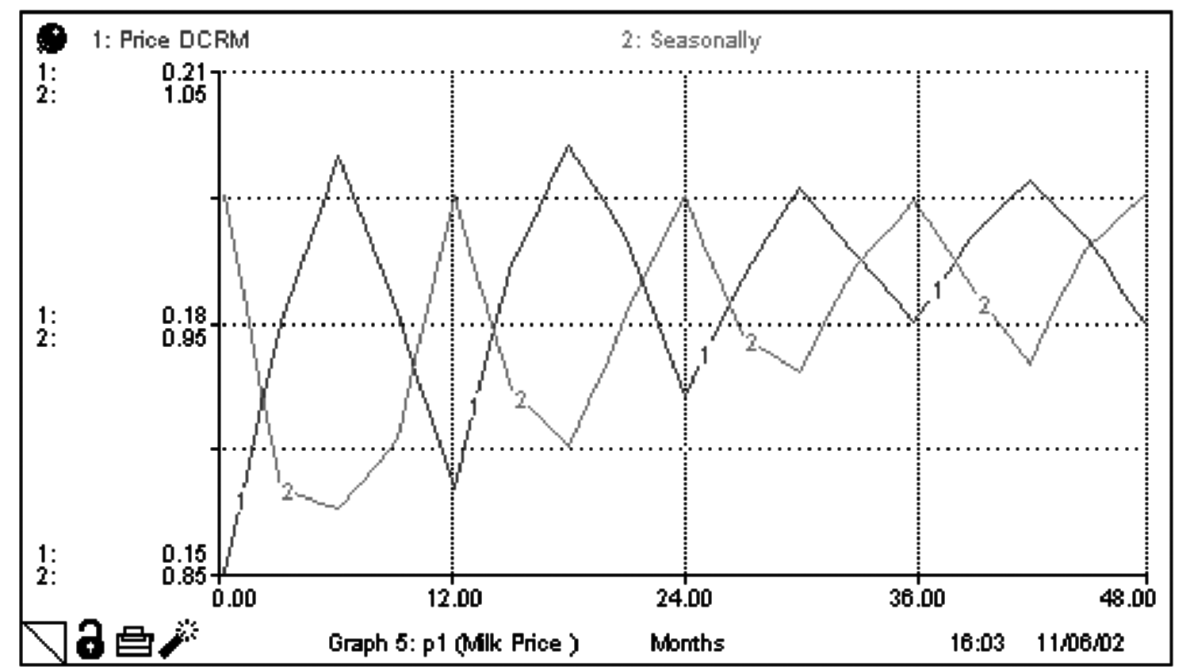

Figure 1. Projected milk price ("1: Price DCRM") and expected seasonality influence ("2: Seasonality") over the simulation time. 
cost ("VcuRMDC"), which is the price paid for the milk supplied "Price DCRM", other variable unit costs ("Other $\mathrm{Vc} \mathrm{DC}^{\prime \prime}$ ), and the respective part of the network technical assistance cost ("DCTAcu"). Fixed costs and the variable unit cost are based on the real cost structure of a mediumscale dairy processor in Brazil's southeast region.

The difference between the price paid by the dairy company and the rural producers' total unit cost over the simulation time is the unit profit margin of each group of farms ("Profit1u", "Profit $2 u$ ", and "Profit $3 u^{\prime \prime}$ ). It is noteworthy that the unit profit margin is the difference between the cost of purchase or production and the selling price. Thus, this profit margin can occur as a negative value. In this case, dairy farms will face a non-profitable operational result. Appendix B lists the equations and the input data that we have chosen for the demonstration of the full model.

Other assumptions for two important model variables were made to construct alternative scenarios. Three scenarios were developed to run the proposed model and were based on the preliminary studies by Novaes et al. (2002) from a governmental agency for agricultural development. These authors studied for three years the impacts of adequate sanitary control of the animals, pasture improvements and technological innovations on the farm's milk production. In the current study, all simulations were run for 48 months. Thus, we foresaw a gradual improvement in farm productivity. Three assumptions were made in terms of expected performance of the productivity improvement rate and refusal rate over time. Scenario 1 represents a moderate expected performance of the coordination agent while scenarios 2 and 3 represent, respectively, an optimistic and pessimistic projection. First, productivity improvements are projected by each group of rural producers at the $8^{\text {th }}$ month of each simulation through a "time" function in the model (impacts of technical assistance actions). Second, the simulation projects a gradual reduction of the refusal rate ("Refusal rate" in the model) from the $12^{\text {th }}$ month. These two variables in scenarios 2 and 3 are projected to have more and less impact on the system with rates oscillating around the moderate scenario. Figures 2(a), 2(b), and 2(c) show the projections respectively for scenarios 1,2 , and 3 .

The projected behaviors shown in the moderate scenario (scenario 1) represent a very reasonable waste reduction in the system over time after the coordination agent's actions (e.g., sanitary control of animals, appropriate milking techniques, etc.) have started in the whole network. These quantity and quality behaviors are considered in this study to be an average potential for the whole network. Of course, some dairy farms have more potential to develop the projected results, depending on their production capacity and initial technological condition.
It is important to emphasize that the improvement in the refusal and technical assistance rates shown in Figure 2 projects the reduction of the waste of the system and the improvement of the initial low rate of farm productivity.

\section{Results}

The main expected result was that the agents' cost would be reduced by diluting fixed costs. However, the actions to be performed by the technical assistance agent can also reduce some total variable and fixed costs. For example, the intensive biological and chemical tests carried out at the dairy company's reception plant to verify the milk quality can be reduced when the raw material reliability is improved. Thus, labor and material expenses spent to perform such activities can be reduced. In view of costs, these are not value-added activities. Therefore, they should be eliminated or minimized to increase the performance of the global system. This possible cost reduction is not foreseen in this study. Thus, total fixed costs and unit variable costs (input data) are constant over the simulation time, except for the technical assistance costs that are incorporated into the system at the $6^{\text {th }}$ month of simulation.

It was expected that the production costs would fall over the simulation time after the incorporation of the policies and actions proposed in the model: first, through larger supply regularity and second, through better quality of the raw material. As a result, a production unit cost reduction for each group of rural producers is foreseen over the simulation time (48 months).

Similarly, the dairy company can reduce its unit production cost ("TcuDC") by increasing the total volume processed ("DC processing" in the "Dairy Co." sector in the model). Figures 3(a), 3(b), and 3(c) show respectively for scenarios 1,2 , and 3 the three rural producer groups and the dairy company unit production cost foreseen over the simulation time. The unit cost of each group of farms and the dairy company increases at the $6^{\text {th }}$ month. This is a result of the combination of the first simulated dry season (lower outcome) and the incorporation of the "F1Tac", "F2Tac", "F3Tac", and "DCTAc" costs.

However, the effective milk flow over time for each group of rural producers increases over the simulation time, reducing the fixed and technical assistance unit costs of each group of farms. In the same way, the increase in the monthly volume of milk processed reduces fixed and technical assistance unit costs of the dairy company.

The difference between the initial and final costs depends on the assumptions projected in each scenario. With an optimistic approach scenario 2 has the greatest and fastest decrease in agent's production unit cost over the simulation time (Figure 3(b)). 

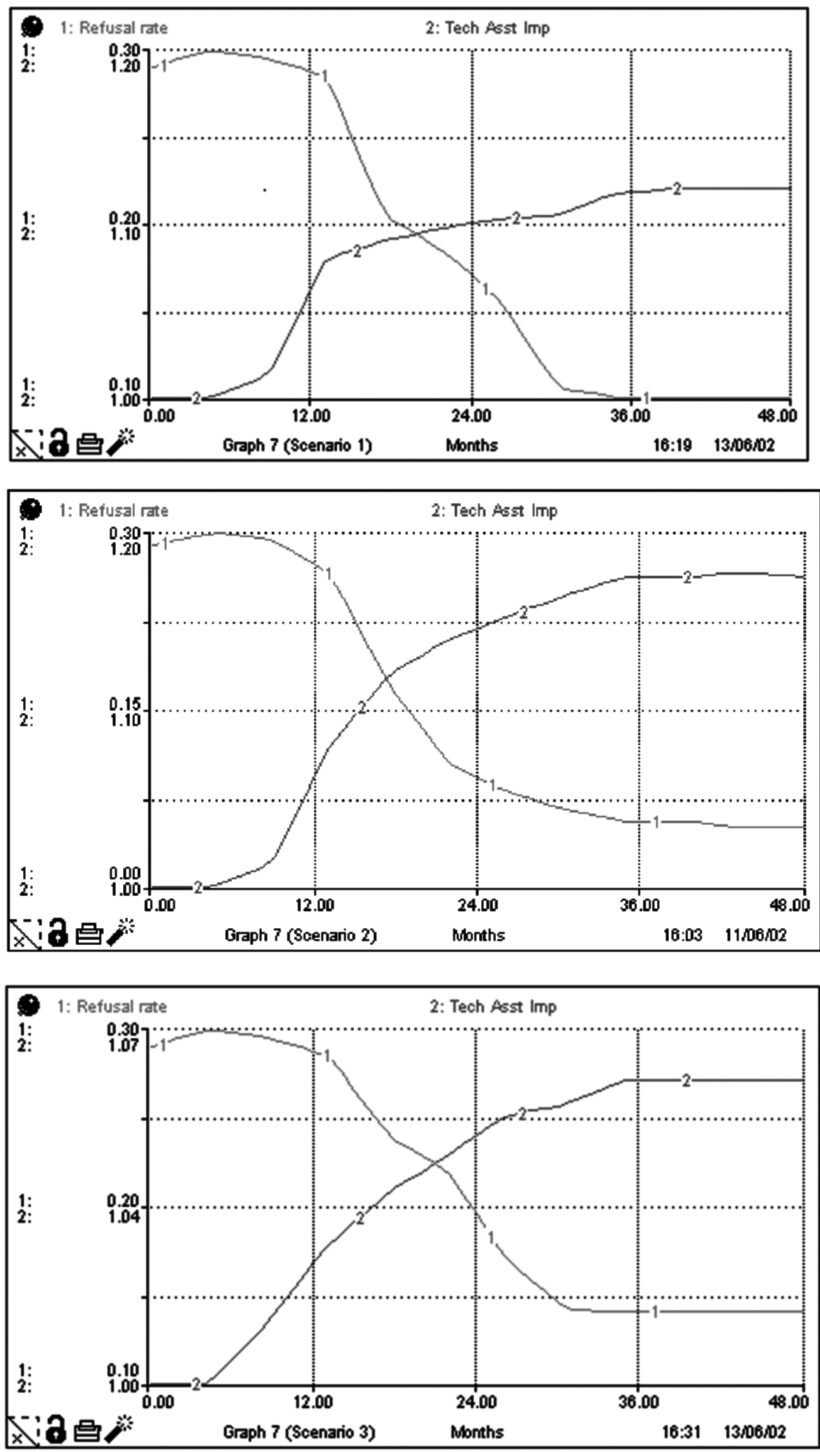

Figure 2. Refusal rate ("1: Refusal rate") and technical assistance improvement ("2: Tech Asst Imp") assumptions: a) Moderate scenario 1; b) Optimistic scenario 2; c) Pessimistic scenario 3.

As a direct consequence of the results cited above, more attractive or non-negative operational margins (profit margin) are expected for the rural producers when production costs fall over time. The same can be expected for the dairy company when its processing cost also falls. In this study, however, we only evaluated the profit margins of the three groups of rural producers represented in the model. The dairy company's profit margin depends on its market performance compared with its cost (operational) performance. This operational performance is indeed more crucial for the dairy company because the raw material cost represents around $45 \%$ of the total in its cost structure. Therefore, in the Brazilian dairy industry the leverage point seems to be the effective management of the supply chain. An analysis of the dairy company's market performance would be beyond the scope of this paper.

As Figures 4(a), 4(b), and 4(c) show, the profit margin for the third group of rural producers is negative at the first summer (current situation); i.e., this group of rural producers is initially facing non-profitable operational results. With the moderate approach scenario 1 , it is forecasted that the small-scale group of farms can achieve the break-even point (cost and revenue equivalence) only at the $15^{\text {th }}$ month of the simulation (after the second summer season). This shows that these small-scale rural producers are more vulnerable in absorbing new costs. 

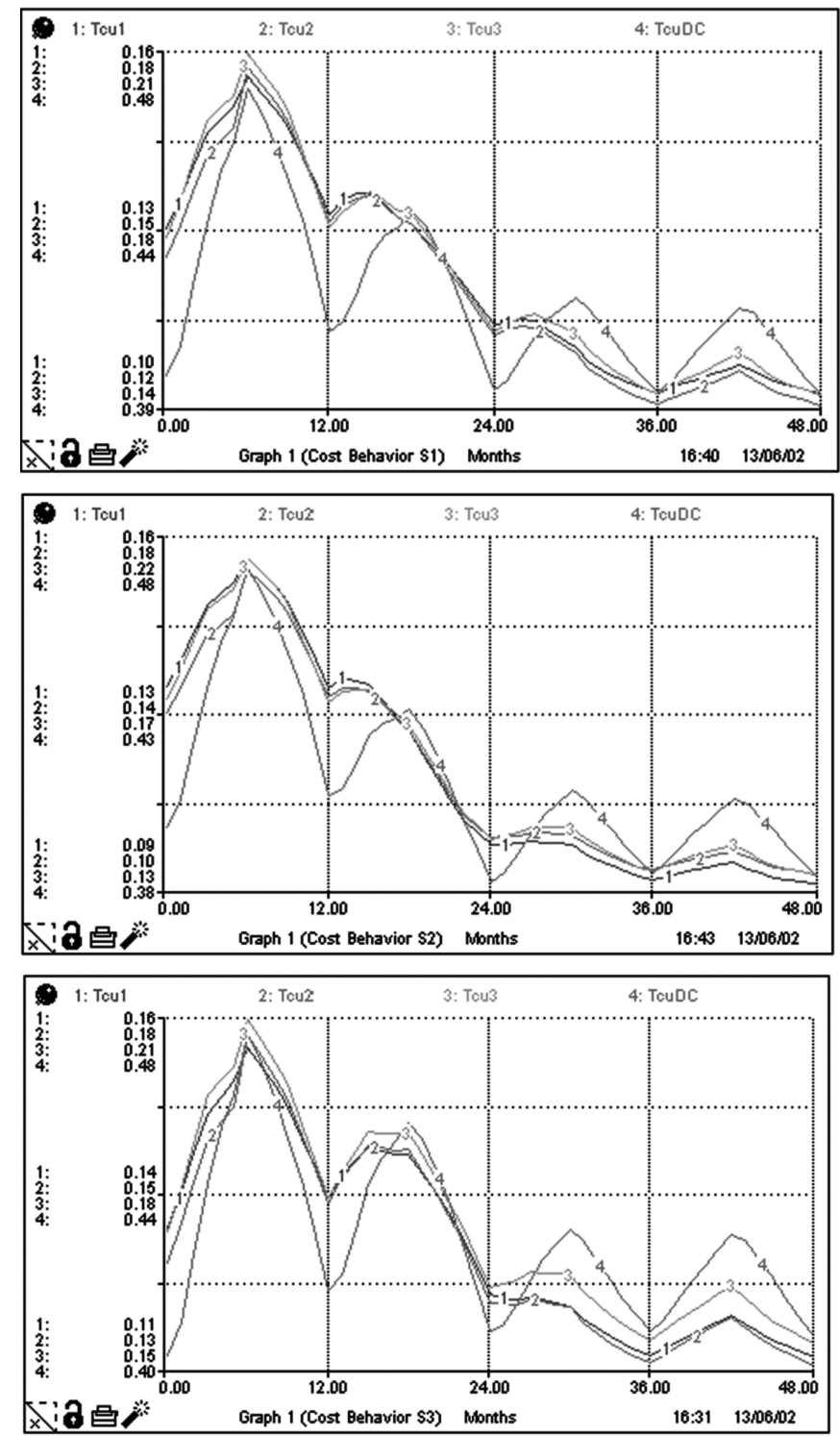

"Tcu1" - medium-scale producers' production cost; "Tcu2" - large-scale producers' production cost; "Tcu3" - small-scale producers' production cost; "TcuDC" - dairy company's production cost

Figure 3. Forecasted unit production cost for the three farm groups and for the dairy company: a) scenario 1 ; b) scenario 2 ; c) scenario 3.

Nevertheless, the significant result of the incorporated policies throughout the system is the incremental change of the rural producers' profit margin, regardless of which scenario is considered in the analysis. This is because the agents' quantity and quality performance are projected to improve and simultaneously the price oscillation is projected to be less strong. Of course, for the optimistic scenario (Figure 4(b)) the profit margins are forecasted to quickly increase for the first and second group of farms. Again, the small-scale group of farms can reach the break-even point at the $14^{\text {th }}$ month; i.e., right after the second simulated summer season.

However, even if we consider the pessimistic approach of scenario 3 (Figure 4(c)), the small-scale dairy farms can reach a positive operational result close to the second simulated dry season ( $17^{\text {th }}$ month). Although it is not the most desired situation, there is hope for the small and low- 

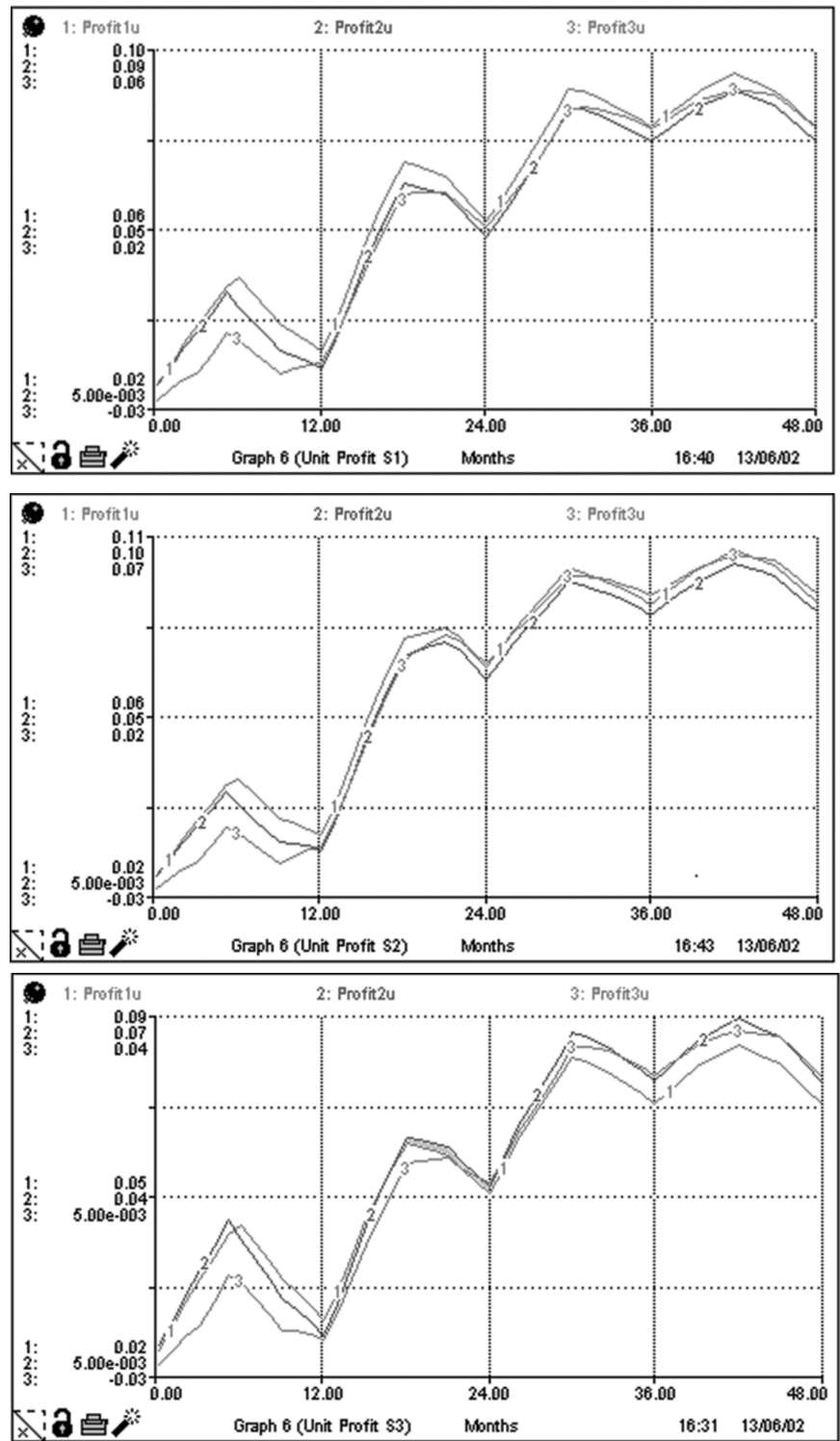

"Profit1u" - medium-scale producers' profit margin; "Profit2u" - large-scale producers' profit margin; “Profit3u" small-scale producers' profit margin;

Figure 4. Forecasted unit profit for the three farms groups over the simulation time: a) scenario 1; b) scenario 2; c) scenario 3.

productive dairy farms. Even with a pessimistic approach, these rural companies could survive by themselves after receiving public or private technical and administrative support.

\section{Summary and discussion}

The application of the concepts of SCM is considered to be an alternative to improve agrichains performance. The use of SD simulation can be very useful in the attempt to validate these concepts and identify and achieve the projected benefits. The proposed SD simulation model can help agribusiness managers find a way to measure and report costs and other data that span company boundaries, share information about the level of the benefits and with which members they reside, and reallocate the benefits that put some supply chain members in a worse position as a result of their cooperation. 
The projections for each of the scenarios given and the input data that we have chosen for the demonstration of the full model are estimated and based on literature, the dairy company and the cost structure of its rural producers' network in Brazil's southeast region. The model was explained to the dairy company's managers, who considered it to be a very adequate management tool. They pointed out that the proposed model (and other models that can be derived from the proposed one) could be very useful for the decision-making process in SCM. As a result, they have created the position of "Supply Chain Manager" in the dairy company's staff. Unfortunately, the majority of the dairy companies in Brazil and the dairy company and its network of rural producers that we selected as a reference do not have historical data for all the model variables. Indeed, they can neither collect data nor monitor all the variables set in the proposed SD model.

Collecting the historical and real data is a long-term activity, during which the model can also work as feedback and a control mechanism of the joint actions taken by the supply chain economic agents. The final results, of course, depend on the assumptions. In the absence of adequate data particularly regarding the technical assistance improvements in the farms' productivity and the refusal rate reduction several assumptions had to be made for this study. However, it is possible to test alternative specifications within the proposed SD framework. In fact, the great advantage is that it readily permits examination of a wide range of alternative models and scenarios (Smith and van Ackere, 2002).

Indeed, supply chain agents' decision-making processes can be facilitated when the policies' impacts are previously known. The application of SD modeling also shows a high degree of applicability as a management tool in the reformulation process of public and/or private policies seeking to improve the efficiency of economic and productive systems (agricultural supply chains). The great advantage of this method is that we can put a very complex problem, which may require many pages of narrative explanation, onto a small set of graphical presentations.

However, correct understanding of the problem, and the correct definition of the variables and the information network are essential in taking full advantage of the SD method. By using the SD approach it is possible to recognize and take advantage of the leverage that can be gained by the effective management of the supply chain.

The model developed in this paper facilitates strategic analysis of productive systems in today's complex global business world. It is expected to improve forward supply chain decision making while evaluating new interventionist policies for more efficient performance.

\section{Acknowledgments}

We thank the anonymous referees for the suggestions given to the work. We also thank Professor Shijie Deng for discussing supply chain modeling principles with us. We appreciate the attention given to the English revision by Patricia Zahra. CAPES - Government of Brazil - have supported the preparation of this paper and the research that it describes.

\section{References}

Anderson, V and Johnson, L., 1997. Systems Thinking Basics: From Concepts to Casual Loops. Pegasus Communications, Inc., Cambridge, MA.

Ballou, R.H., Gilbert, S.M. and Mukherjee, A., 2000. New managerial challenges from supply chain opportunities. Industrial Marketing Management, v. 29, 7-18.

Buongiorno, J., 1996. Forest sector modeling: a synthesis of econometrics, mathematical programming, and system dynamics methods. International Journal of Forecasting, v. 12, 329-343.

Cooper, M.C., Lambert, D.M. and Pagh, J. D., 1997. Supply Chain Management: More Than a New Name for Logistics. The International Journal of Logistics Management, v. 8, n. 1, 1-13.

Cover, J., 1996. Introduction to System Dynamics. Powersim Press, Sterling, VA.

Fisher, D.K., Norvell, J., Sonka, S., Nelson, M.J., 2000. Understanding technology adoption through system dynamics modeling: implications for agribusiness. International Food and Agribusiness Management Review, v. 3, 281-296.

Horngren, C.T. and Foster, G., 1991. Cost Accounting: A Managerial Emphasis. Prentice-Hall International, London.

Leite Brasil, CNA/Decon, Embrapa, 2000. Classificação de empresas/marcas - Base de dados 2000 (http://www.leitebrasil.org.br).

Novaes, N.J., Camargo, A.C. de, Manzano, A., Esteves, S.N., Freitas, A.R. de, and Machado, R., 2002. Avaliação de Tecnologias Agropecuárias em Estabelecimentos Familiares com Produção de Leite no Município de São Carlos/SP. Congresso Brasileiro de Veterinária, Embrapa Pecuária Sudeste (Brazilian Conference on Veterinary Science, Viscosa/MG)

Saito, J.R., 2000. Análise de Cadeias Agroindustriais Utilizando Simulação Computacional Baseada na Metodologia de System Dynamics: Um Estudo de Caso. Dissertação de mestrado, Departamento de Engenharia de Produção (Master Degree Thesis, Production Engineering Department) , UFSCar.

Silva, J.F., 2000. Brazil Dairy and Products Annual 2000. Foreign Agricultural Service - Global Agriculture Information Network - Required Report, United States Government.

Senge, Peter M. et al., 1994. The Fifth discipline fieldbook: strategies and tools for building a learning organization. Currency, Doubleday, New York. 
Smith, P.C. and van Ackere, A., 2002. A note on the integration of system dynamics and economic models. Journal of Economic Dynamics \& Control, v. 26: 1-10.

Sonka, S.T. and Cloutier, L. M., 1998. System Dynamics to Evaluate Information Coordination in Agricultural Supply Chains. Revista Brasileira de Agroinformática, v.1, n.1: 1-16.
Stella and Stella Research, 1997. An Introduction to System Thinking. (c)1992-1997 High Performance Systems, Inc.

Yonenaga, W.H., 2001. Utilização de System Dynamics no gerenciamento de florestas plantadas. Dissertação de mestrado, Departamento de Engenharia de Produção, UFSCar.

\section{Appendix A: Model Structure}

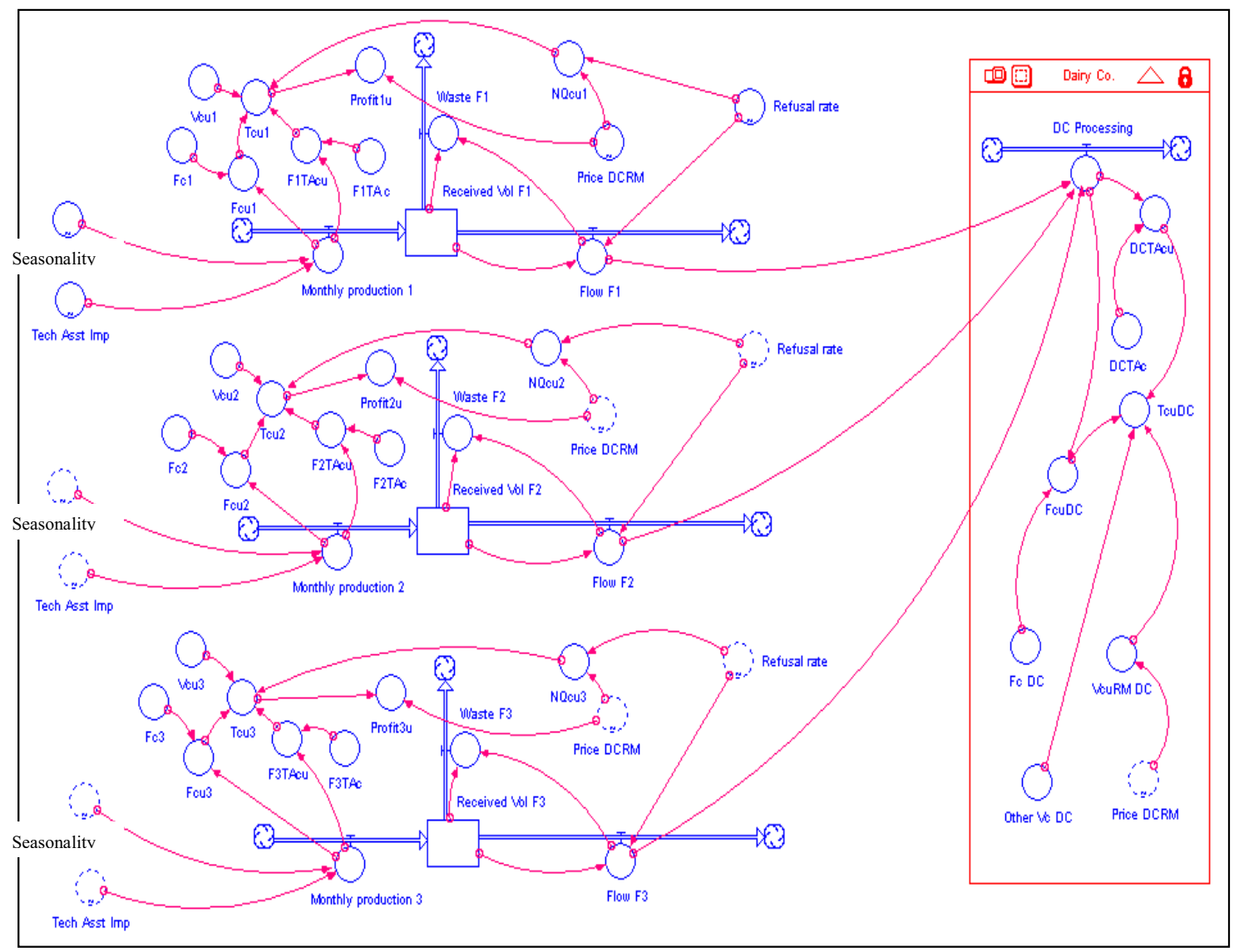

\section{Legend}

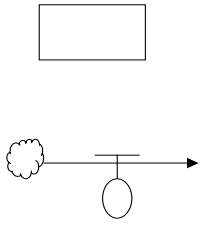

Stocks; physical or abstract accumulations

Flows; things in motion in the direction indicated by the arrowhead (conserved flows drain one stock as they fill another. Non-conserved flows use clouds, which indicate model boundary: a source cloud of the system indicates that for the purpose of a model, an "unlimited" supply of the stuff is assumed to exist; a sink cloud indicates that an "unlimited" capacity exists for absorbing the flow).

Converters; to represent either information or quantities (the value of a converter is re-calculated in each round that calculations are performed)

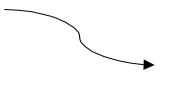

Connectors; network information flow (the relationship among the elements of the model) 


\section{Appendix B: Specification of the model}

\author{
Dairy Co. \\ UNATTACHED: \\ DC_Processing $=$ Flow_F1+Flow_F2+Flow_F3 \\ DCTAc $=$ DELAY $(10000,6,0)$ \\ DCTACu $=$ DCTAC $/$ DC_Processing \\ $\mathrm{FcuDC}=$ FC_DC/DC_Processing \\ FC_DC $=200000$ \\ Other_Vc_DC $=0.12$ \\ TcuDC $=$ FcuDC+Other_Vc_DC+VcuRM_DC+DCTAcu \\ VcuRM_DC $=$ Price_DCRM \\ Not in a sector \\ Received_Vol_F1 $(\mathrm{t})=$ Received_Vol_F1 $(\mathrm{t}-\mathrm{dt})+$ \\ (Monthly_production_1 - Flow_F1 - Waste_F1) ${ }^{*} \mathrm{dt}$ \\ INIT Received_Vol_F1 = 1182000 \\ INFLOWS: \\ Monthly_production_1 = \\ $500 * 80 * 30 *$ Seasonality*Tech_Asst_Imp \\ OUTFLOWS: \\ Flow_F1 = Received_Vol_F1*(1-Refusal_rate) \\ Waste_F1 = Received_Vol_F1-Flow_F1 \\ Received_Vol_F2 $(\mathrm{t})=$ Received_Vol_F2 $(\mathrm{t}-\mathrm{dt})+$ \\ (Monthly_production_2 - Flow_F2 - Waste_F2) ${ }^{*} \mathrm{dt}$ \\ INIT Received_Vol_F2 $=445000$ \\ INFLOWS: \\ Monthly_production_2 = \\ $1000 * 15 * 30 *$ Tech_Asst_Imp*Seasonality \\ OUTFLOWS: \\ Flow_F2 $=$ Received_Vol_F2* $*$ 1-Refusal_rate $)$ \\ Waste_F2 = Received_Vol_F2-Flow_F2 \\ Received_Vol_F3(t) = Received_Vol_F3 $(\mathrm{t}-\mathrm{dt})+$ \\ (Monthly_production_3 - Flow_F3 - Waste_F3) * dt \\ INIT Received_Vol_F3 = 575000
}

INFLOWS:

Monthly_production_3 =

$100 * 200 * 30 *$ Seasonality*Tech_Asst_Imp

OUTFLOWS:

Flow_F3 = Received_Vol_F3*(1-Refusal_rate)

Waste_F3 = Received_Vol_F3-Flow_F3

$\mathrm{F} 1 \mathrm{TAC}=$ DELAY $(3333.34,6,0)$

F1TAcu = F1TAc/Monthly_production_1

$\mathrm{F} 2 \mathrm{TAC}=\operatorname{DELAY}(3333.34,6,0)$
F2TAcu = F2TAc/Monthly_production_2

F3TAC $=$ DELAY $(3333.34,6,0)$

F3TAcu $=$ F3TAc/Monthly_production_3

$\mathrm{FC} 1=700 * 80$

$\mathrm{FC} 2=1700 * 15$

$\mathrm{FC} 3=300 * 200$

Fcu 1 = Fc1/Monthly_production_1

Fcu2 = Fc2/Monthly_production_2

Fcu3 = Fc3/Monthly_production_3

NQcu1 $=$ Refusal_rate* Price_DCRM

NQcu2 $=$ Refusal_rate ${ }^{*}$ Price_DCRM

NQcu3 = Refusal_rate* ${ }^{*}$ Price_DCRM

Profit $1 \mathrm{u}=$ Price_DCRM-Tcu 1

Profit2u = Price_DCRM-Tcu2

Profit $3 \mathrm{u}=$ Price_DCRM-Tcu3

$\mathrm{Tcu} 1=\mathrm{Fcu} 1+\mathrm{Vcu} 1+\mathrm{NQcu} 1+\mathrm{F} 1 \mathrm{TAcu}$

$\mathrm{Tcu} 2=\mathrm{Fcu} 2+\mathrm{NQcu} 2+\mathrm{Vcu} 2+\mathrm{F} 2 \mathrm{TAcu}$

Tcu3 = Fcu3+NQcu3+Vcu3+F3TAcu

$\mathrm{Vcu} 1=0.04$

$\mathrm{Vcu} 2=0.04$

$\mathrm{Vcu} 3=0.03$

Price_DCRM = GRAPH(TIME)

$(0.00,0.15),(3.00,0.18),(6.00,0.2),(9.00,0.181)$,

$(12.0,0.16),(15.0,0.187),(18.0,0.201),(21.0,0.19)$,

$(24.0,0.171),(27.0,0.185),(30.0,0.196),(33.0,0.188)$,

$(36.0,0.18),(39.0,0.19),(42.0,0.197),(45.0,0.19)$,

$(48.0,0.18)$

Refusal_rate $=$ GRAPH $($ TIME $)$

$(0.00,0.29),(4.36,0.3),(8.73,0.295),(13.1,0.265)$,

$(17.5,0.17),(21.8,0.105),(26.2,0.08),(30.5,0.065)$,

$(34.9,0.055),(39.3,0.055),(43.6,0.05),(48.0,0.05)$

Seasonality $=$ GRAPH(TIME)

$(0.00,1.00),(3.00,0.883),(6.00,0.875),(9.00,0.903)$,

$(12.0,1.00),(15.0,0.923),(18.0,0.9),(21.0,0.953)$,

$(24.0,1.00),(27.0,0.945),(30.0,0.93),(33.0,0.973)$,

$(36.0,0.998),(39.0,0.965),(42.0,0.933),(45.0,0.98)$,

(48.0, 1.00)

Tech_Asst_Imp $=$ GRAPH(TIME)

$(0.00,1.00),(4.36,1.00),(8.73,1.01),(13.1,1.08)$,

$(17.5,1.12),(21.8,1.14),(26.2,1.15),(30.5,1.17)$,

$(34.9,1.18),(39.3,1.18),(43.6,1.18),(48.0,1.18)$. 